ESJ Natural/Life/Medical Sciences

\title{
Hombres Universitarios: lo que hacen por su salud
}

\author{
José Luis Luna-González, (Estudiante Pasante de la Licenciatura en \\ Enfermería)
}

Diana Alejandra Reséndiz-Guerrero, (Estudiante Pasante de la

Licenciatura en Enfermería)

Sarket Gizeh Juárez-Nilo, (Licenciada en Enfermería.)

Adriana Martínez-Díaz, (Maestra en Tecnología de los Alimentos.)

Facultad de Enfermería, Universidad Autónoma de Querétaro, México

Daniel Arceo Mariñelarena, (Maestro en Administración.)

Universidad Marista de Querétaro, Mexico

Ruth Magdalena Gallegos-Torres, (Doctora en Ciencias de la Salud.)

Facultad de Enfermería, Universidad Autónoma de Querétaro, México

Doi:10.19044/esj.2021.v17n7p243

Submitted: 21 May 2020

Accepted: 22 January 2021

Published: 28 February 2021
Copyright 2021 Author(s)

Under Creative Commons BY-NC-ND

4.0 OPEN ACCESS

Cite As:

Luna-González J.L., Reséndiz-Guerrero D.A., Juárez-Nilo S.G., Martínez-Díaz A., Mariñelarena D.A. \& Gallegos-Torres R.M. (2021). Hombres Universitarios: lo que hacen por su salud. European Scientific Journal, ESJ, 17(7), 243.

https://doi.org/10.19044/esj.2021.v17n7p243

\section{Resumen}

Introducción: Con respecto a la salud, existen diferencias entre hombres y mujeres, además de que los programas de salud están mayormente dirigidos a la mujer. Objetivo: Valorar el autocuidado de la salud en varones universitarios de dos universidades privadas de la ciudad de Querétaro, Qro. Metodología: Investigación transversal, descriptiva; se aplicó un cuestionario a 194 alumnos, de 46 items integrados en 7 dimensiones. Para el análisis de los datos se utilizó el software SPSS v.20 utilizando estadística descriptiva. La investigación fue evaluada y aprobada por un comité de investigación y bioética. Se respetaron los principios bioéticos de investigación. Resultados: Alumnos de 18 a los 32 años. 96\% son solteros. 81.7\% saben qué es el autocuidado. $70.8 \%$ realiza ejercicio por lo menos 30 minutos tres veces a la semana. $49.2 \%$ no conoce que existe cáncer de mama en los varones. $31.7 \%$ consume alcohol cada fin de semana, $35 \%$ ha consumido marihuana y $11 \%$ cocaína en el último año. $84 \%$ son activos sexualmente. En relación al sueño 
y el descanso, el $82 \%$ duerme de 3 a 7 horas diarias. Conclusiones: Los datos globales demuestran que es necesario que los varones refuercen las actividades de autocuidado personal en diversas áreas.

Palabras clave: Hombres, salud del hombre, enfermería

\title{
University Men: what they for their Health
}

\section{José Luis Luna-González, (Estudiante Pasante de la Licenciatura en Enfermería) \\ Diana Alejandra Reséndiz-Guerrero, (Estudiante Pasante de la Licenciatura en Enfermería) \\ Sarket Gizeh Juárez-Nilo, (Licenciada en Enfermería.)} Adriana Martínez-Díaz, (Maestra en Tecnología de los Alimentos.) Facultad de Enfermería, Universidad Autónoma de Querétaro, México Daniel Arceo Mariñelarena, (Maestro en Administración.)

Universidad Marista de Querétaro, Mexico

Ruth Magdalena Gallegos-Torres, (Doctora en Ciencias de la Salud.) Facultad de Enfermería, Universidad Autónoma de Querétaro, México

\begin{abstract}
Introduction: Regarding to health, there are differences between men and women, and health programs are mostly aimed to women. Objective: to evaluate male health self- care in university students of two private universities in Queretaro, city. A cross sectional study was made. A 46 items questionnaire integrated in 7 dimensions was applied to 194 students. The software SPSS v. 20 was used to analyze data using descriptive statistics. The research was evaluated and approved by a research and bioethics committee respecting the research Bioethical principles. Results: Students aged 18 to 32 . $96 \%$ are single. 81.7 know what self-care is. $70.8 \%$ exercise at least 30 minutes thrice a week. $49.2 \%$ do not know there is male breast cancer. $31.7 \%$ consume alcohol every weekend, $35 \%$ have tried marijuana and $11 \%$ cocaine in the last year. $84 \%$ are sexually active. Related to sleep and rest, $82 \%$ sleep 3 to 7 hours a day. Conclusions: Global data shows that it is necessary to reinforce male personal self-care activities in severe areas.
\end{abstract}

Keywords: Men, Men's Health, Nursing

\section{Introduction}

A lo largo de la historia, el concepto de autocuidado se ha ido modificando. Tiene sus orígenes en la cultura griega, en donde se empezó a 
señalar el significado de cuidarse a sí mismo. Los griegos tenían una visión integral de cuidar no sólo el cuerpo sino también el alma, todo esto basado en el principio de cuidarse y respetarse como persona (Uribe J, 1999).

$\mathrm{Al}$ respecto, el autocuidado está ligado fuertemente a la salud, ya que la manera en que la persona cuide de sí misma influirá en el nivel de salud que posea o la percepción que de esta se tenga. Al respecto, la Organización Mundial de la Salud (OMS, 1946) define a la salud como "un estado completo de bienestar físico, mental y social de una persona y no sólo la ausencia de enfermedad", por lo que considera que esta es un derecho fundamental de todo ser humano, lo que incluye su acceso a servicios de calidad, la libertad de controlar su salud sin injerencias, entre otros, para que tenga la oportunidad de alcanzar el grado máximo o idóneo.

Si todos los individuos sin excepción tienen derecho a la salud, esto incluye también a los estudiantes universitarios, quienes transitan de un estado de adolescencia a adultez en pleno ingreso a los estudios profesionales, en donde viven múltiples cambios físicos, emocionales, sociales, y de diversa índole.

La salud del estudiante universitario se ve afectada por diversas condiciones, como pueden ser los trastornos de ansiedad, estrés académico, tensión asociada a situaciones en las que el estudiante requiere retener una determinada cantidad de información en un determinado tiempo y muchas otras similares, así como la insatisfacción social, dificultades económicas y familiares (Caballero D, Abello LI, \& Palacio S, 2018), consumo de alcohol y drogas y muchas más.

De acuerdo a lo anterior, se plantea que la salud del universitario abarca todos los ámbitos del ser humano, de los cuales cabe destacar que existe diferencia entre hombres y mujeres. Socialmente se reconoce que el varón presenta una actitud no favorable sobre pedir ayuda, muestra una negación de la enfermedad y la falta de medidas de autocuidado tanto médicas como las que tienen que ver con el estilo de vida, mientras que la mujer en su mayoría de las veces presenta todo lo contrario con respecto a las actitudes de los hombres (De Keijzer, 1997).

$\mathrm{Al}$ respecto, se reconoce que el género juega un papel importante en los determinantes de salud, los que a su vez están influenciados por el autocuidado que la persona ejerce hacia sí misma. El aspecto complejo en este sentido es la falta de información que tienen las organizaciones sobre los fenómenos en salud por género y que influiría en dichos determinantes (Pinto et al., 2019). De la misma manera, en el cuidado de la salud desde el punto de vista individual, juegan un papel relevante la educación, la cultura, el acceso a los servicios de salud, entre otros.

El autocuidado de la vida y la salud implican autodeterminación. El joven, en el medio universitario, se debe de enfrentar a una diversidad de 
experiencias culturales y generacionales que le obligan a elegir cursos de acción relacionados con factores protectores o de riesgo enfocados al cuidado (Franco Peláez \& Duque Escobar, 2015).

Al no existir una amplia variedad de estudios específicos del autocuidado en salud en los varones universitarios, se crea una demanda por la falta de información para la conservación y reforzamiento de la calidad en salud en esta etapa de la vida, teniendo como consecuencia una esperanza de vida menor y programas de salud sin enfoque a cada género o bien, cuando hay programas, la población desconoce de ellos y no se sabe el impacto que estos tengan en los receptores, cuáles son sus áreas de oportunidad, entre otros, que dificultad el tener sustento para el trabajo en cualquier grupo poblacional.

Diez y nueve años después de iniciado el siglo XXI, la imagen del varón basada en el poder y la potencia aún prevalece. Los varones deben ser activos, "tener el control, estar a la defensiva, ser fuertes, aguantar el dolor, valerse por sí mismos, usar el cuerpo como herramienta, no pedir ayuda, salir adelante, preocuparse por el hacer y no el sentir", no tener empatía, entre otras cosas (Cubillas Rodríguez et al., 2016).

Así mismo, los roles y creencias se adquieren por un proceso de aprendizaje que vincula las relaciones con otros así como de la familia, lo que determina la identidad, lo que se debe hacer o sentir respecto a algo, incluso lo que se debe interpretar, actuar y pensar de la realidad. En este sentido, se puede apreciar que los elementos antes señalados influyen en múltiples aspectos, entre los que figura la salud (Cubillas Rodríguez et al., 2016).

En el informe de la Organización Panamericana de la Salud "Comprender la salud de los hombres" (Sabó, 2000), se expresa que algunos comportamientos de riesgo para la salud son evidentemente "masculinos", de manera tal que así los varones expresan su virilidad. Aunque de la misma manera se reconoce que no todos los hombres son iguales.

Relacionado con lo anterior, algunos resultados de estudios muestran dos aspectos: pocas son las investigaciones realizadas exclusivamente en varones y las que hay abordan pocos aspectos de salud. En este sentido se aprecia que a pesar de que los jóvenes tienen un concepto hacia el autocuidado, ello no se ve reflejado en la realización de la práctica de su autocuidado en el aspecto físico, poniendo en riesgo su estado de salud. Una mayoría relativa de adolescentes realizan prácticas de autocuidado adecuadas en cuanto a su aspecto social y psicológico (Quinchiguano Caraguay, 2017).

En una investigación realizada en Brasil con 534 estudiantes universitarios, se encontró que el $75.2 \%$ de los hombres no participó en actividades relacionadas con el cuidado de la salud en lo referente al consumo de tabaco, dieta, ejercicio físico u otros en el último año (da Cruz Mendonça et al., 2018). 
Una investigación realizada en una universidad de Chile, con 370 alumnos, de los cuales el $40 \%$ era varón, encontró en ellos que $12.5 \%$ tenía sobrepeso y el 3\% obesidad, $27.9 \%$ fumaban. Los chicos, dormían en promedio 6.25 horas, destacando que aquellos que fuman presentan mayor somnolencia diurna (Guerrero-Wyss, Méndez, Gajardo, \& Durán Agüero, 2018).

Igualmente, se realizó un estudio en 200 universitarios varones de Lima, Perú. Se valoró consumo de alcohol y síntomas de depresión. Los datos indicaron que el $21 \%$ consumía alcohol 1 vez por semana, $7 \% 2$ veces por semana y $2 \%$ de 3 a 6 veces por semana. Con respecto a la depresión, $48.5 \%$ manifestó síntomas de depresión leve, 16\% moderada y 3\% grave (EstradaDurand \& Salinas-Salas, 2019).

En México, no hay estudios específicos sobre el cuidado de la salud en varones, de manera general. Se pueden encontrar, como las investigaciones mostradas, abordajes en temas particulares, sin embargo, más que nada separan al grupo de hombres y mujeres y se muestran los resultados de ambos.

Por tanto, con base a lo anterior, esta investigación resalta la necesidad de realizar una aproximación a la valoración del autocuidado de la salud en varones universitarios de dos instituciones privadas de la ciudad de Querétaro: la Universidad Politécnica de Querétaro y la Universidad Marista de Querétaro, buscando las áreas favorables y débiles que impliquen el desarrollo de programas en salud para el mantenimiento de la misma o la prevención.

\section{Metodología:}

Se realizó un estudio con enfoque cuantitativo, de tipo transversal, descriptivo y comparativo. El universo lo constituyeron los estudiantes de la Universidad Politécnica de Querétaro (UPQ) y Universidad Marista de Querétaro. La población consistió en un aproximado de 250 alumnos del género masculino de la Universidad Politécnica de Querétaro, mientras que en la Universidad Marista de Querétaro se contaba con una población estudiantil aproximada de 150 alumnos. Por el tamaño reducido de la población se aplicaron los instrumentos a 120 hombres de la Universidad Politécnica de Querétaro y 74 en la Universidad Marista de Querétaro, de las carreras de ingeniería diversas con las que cuenta cada institución. Se excluyeron a mujeres, estudiantes de movilidad y alumnos no regulares, así mismo se eliminaron cuestionarios de alumnos que no contestaron el cuestionario en un 95\% o que marcaron dos respuestas en alguna pregunta.

El muestreo fue por conveniencia, ya que cada institución señaló los tiempos de aplicación de los cuestionarios y los alumnos con los que se trabajaría.

Para la obtención de permisos se efectúo el registro del proyecto ante el Comité de Investigación de la Facultad de Enfermería de la Universidad 
Autónoma de Querétaro; posterior a esto se solicitó el permiso a las instituciones ya señaladas a través de la Coordinación de cada una mediante oficio, donde se especificó el objetivo del estudio, tiempo estimado para la recolección de datos, fecha de aplicación de los cuestionarios, así como las características de este último.

Para la recolección de datos se utilizó un cuestionario para la identificación del autocuidado en varones, elaborado por Bárcenas, Hernández, Hernández y Gallegos (2017), validado y aplicado en 2017 en 200 alumnos de ingeniería de una universidad pública de Querétaro.

El cuestionario inicia con una cédula de datos sociodemográficos que incluye: edad: (años cumplidos), nivel socioeconómico, estado civil, ocupación, religión. Continúa con una sección de 46 ítems, agrupados por dimensiones: Conocimiento de Autocuidado (6 ítems), Cuidado Físico (11 ítems), Actividades extraescolares (3 ítems), Adicciones (6 ítems), Alimentación (5 ítems), Autocuidado sexual (9 ítems), Sueño y descanso (6 ítems). Las opciones de respuesta corresponden a posibilidades dicotómicas, con datos de SI / NO.

Para el análisis estadístico de los datos se utilizo el paquete SPSS (Statistical Package for the Social Sciences) versión 22.0 en español y del programa Microsoft Excel. Así mismo, se utilizó estadística descriptiva a través de frecuencias, media, moda, mediana, desviación estándar y porcentajes para describir el fenómeno estudiado.

Este estudio de investigación tuvo como sustento ético la Ley General de Salud (Cámara de diputados del H. Congreso de la Unión, 1987), Código de Nüremberg (Comité Nacional de Bioética, 1947) y la Declaración de Helsinki (CONAMED, 2001), donde se indica el respeto de los aspectos de la confidencialidad por medio del consentimiento informado y la individualidad.

\section{Resultados:}

A continuación, se presentan los datos sociodemográficos de los alumnos por institución.

\section{Universidad UPQ}

Participaron 120 alumnos universitarios. Las edades de los participantes fueron de los 18 a 32 años, con una media $20.25 \pm 1.82$. El 95.8\% son solteros, $80.8 \%$ se considera en un nivel económico medio. Estudian y trabajan $33.3 \%$. El $87.8 \%$ es de religión católica.

\section{Universidad Marista}

Participaron 74 alumnos, la edad de los participantes abarca desde los 17 a $\operatorname{los} 28$ años, con una media de $21.6 \pm 2.2$. 89.2\% son de nivel 
socioeconómico medio, $93.2 \%$ son solteros, $63.5 \%$ estudian y trabajan, el $87.8 \%$ son católicos.

En la tabla 1, 2 y 3 se pueden apreciar los principales hallazgos en los ítems del cuestionario en los alumnos de ambas universidades.

Tabla 1. Datos sociodemográficos y el conocimiento sobre autocuidado de los estudiantes universitarios

\begin{tabular}{lcc}
\hline Ítems & $\begin{array}{c}\text { Universidad } \\
\text { Politécnica de Querétaro }\end{array}$ & Universidad Marista \\
& & \\
\cline { 2 - 3 } & $\mathrm{Si}$ & $\mathrm{Si}$ \\
\hline Sabes qué es el autocuidado & 81.7 & $91.9 \%$ \\
Calidad de Vida & $15 \%$ & $10.8 \%$ \\
Aseo personal & $6.7 \%$ & $8.1 \%$ \\
Acciones generales & $21.7 \%$ & $14.9 \%$ \\
Acciones especificas & $56.7 \%$ & $66.2 \%$
\end{tabular}

¿Qué áreas consideras que un hombre debe cuidar?

\begin{tabular}{lcc}
\hline Área Física & $90.8 \%$ & $95.9 \%$ \\
Área Emocional & $84.2 \%$ & $81.1 \%$ \\
Área Sexual & $86.7 \%$ & $93.2 \%$ \\
Área Económica & $60.8 \%$ & $60.8 \%$ \\
Área Escolar & $64.2 \%$ & $62.2 \%$ \\
Área Personal & $89.2 \%$ & $93.2 \%$ \\
Área Relaciones Sociales & $66.7 \%$ & $66.2 \%$ \\
Área Estado de Salud & $89.2 \%$ & $89.2 \%$ \\
Área Alimentación & $88.3 \%$ & $93.2 \%$ \\
Área Familiar & $75.8 \%$ & $73 \%$ \\
\hline
\end{tabular}

Nota: la tabla muestra información general vinculada al tema, de manera tal que se obtuvo una primera apreciación de los jóvenes sobre el fenómeno. 
Tabla 2. Hábitos saludables y nocivos de los estudiantes

\begin{tabular}{|c|c|c|}
\hline Ítems & $\begin{array}{c}\text { Universidad Politécnica de } \\
\text { Querétaro }\end{array}$ & Universidad Marista \\
\hline Ejercicio 30min & $70.8 \%(\mathrm{Si})$ & $68.9 \%(\mathrm{Si})$ \\
\hline Ejercicio 3 veces $\mathrm{x}$ semana & $57.5 \%(\mathrm{No})$ & $56.8 \%(\mathrm{Si})$ \\
\hline Acude al médico & $63.3 \%(\mathrm{No})$ & $60.8 \%$ No \\
\hline Chequeo en ultimo año & $57.5 \%(\mathrm{Si})$ & $56.8 \%(\mathrm{Si})$ \\
\hline Bloqueador todos los días & $85.8 \%$ (No) & $81.1 \%$ (No) \\
\hline Hidrata piel con cremas & $60.8 \%(\mathrm{Si})$ & $67.6 \%(\mathrm{Si})$ \\
\hline Cuidado de uñas & $79.2 \%(\mathrm{Si})$ & $78.4 \%(\mathrm{Si})$ \\
\hline Aseo después del ejercicio & $95.8 \%(\mathrm{Si})$ & $95.9 \%(\mathrm{Si})$ \\
\hline Automedicación & $55.8 \%(\mathrm{Si})$ & $51.4 \%(\mathrm{Si})$ \\
\hline $\begin{array}{l}\text { Existe cáncer de mama en } \\
\text { hombres }\end{array}$ & $50.8 \%(\mathrm{Si})$ & $58.1 \%(\mathrm{Si})$ \\
\hline Palpación en pectorales & $71.7 \%(\mathrm{No})$ & $77 \%$ (No) \\
\hline Actividades recreación & $97.5 \%(\mathrm{Si})$ & $95.9 \%(\mathrm{Si})$ \\
\hline Platicar con personas & $86.7 \%(\mathrm{Si})$ & $94.6 \%(\mathrm{Si})$ \\
\hline $\begin{array}{l}\text { Actividades de recreación por } \\
\text { semana }\end{array}$ & $89.2 \%(\mathrm{Si})$ & $91.9 \%(\mathrm{Si})$ \\
\hline Cajetilla de cigarros por semana & $87.5 \%(\mathrm{No})$ & $82.4 \%(\mathrm{No})$ \\
\hline Fuman diario & $80 \%$ (No) & $86.5 \%(\mathrm{No})$ \\
\hline Alcohol c/fin de semana & $68.3 \%(\mathrm{No})$ & $51.4 \%(\mathrm{No})$ \\
\hline Alcohol c/tercer día & $91.7 \%(\mathrm{No})$ & $85.1 \%(\mathrm{No})$ \\
\hline Consumo de marihuana & $65 \%(\mathrm{No})$ & $74.3 \%(\mathrm{No})$ \\
\hline Consumo de cocaína & $89.2 \%(\mathrm{No})$ & $86.5 \%(\mathrm{No})$ \\
\hline Alimentación sanamente & $59.2 \%(\mathrm{Si})$ & $50 \%(\mathrm{Si} / \mathrm{No})$ \\
\hline Frituras diariamente & $70 \%$ (No) & $82.4 \%(\mathrm{No})$ \\
\hline Refresco diariamente & $63.3 \%(\mathrm{No})$ & $70.3 \%(\mathrm{No})$ \\
\hline Intervalos de comida & $75.8 \%(\mathrm{Si})$ & $70.3 \%(\mathrm{Si})$ \\
\hline Bebo 2lts al día & $68.3 \%(\mathrm{Si})$ & $64.9 \%(\mathrm{Si})$ \\
\hline Iniciado vida sexual & $84.2 \%(\mathrm{Si})$ & $89.2 \%(\mathrm{Si})$ \\
\hline
\end{tabular}

Nota: La tabla muestra los datos más sobresalientes sobre cada uno de los rubros abordados, separándolos por institución educativa. 
Tabla 3. Sexualidad y descanso de los estudiantes universitarios

\begin{tabular}{lll}
\hline \multicolumn{1}{c}{ Ítems } & $\begin{array}{c}\text { Universidad } \\
\text { Politécnica de } \\
\text { Queretaro }\end{array}$ & $\begin{array}{c}\text { Universidad } \\
\text { Marista }\end{array}$ \\
\hline Uso del preservativo & $61.7 \%(\mathrm{Si})$ & $64.9 \%(\mathrm{Si})$ \\
Más de 2 parejas & $55.8 \%(\mathrm{No})$ & $64.9 \%(\mathrm{Si})$ \\
Conoce enfermedades de Transmisión & $97.5 \%(\mathrm{Si})$ & $94.6 \%(\mathrm{Si})$ \\
Sexual & & \\
Planificación familiar & $50.8 \%(\mathrm{No})$ & $52.7 \%(\mathrm{Si})$ \\
Aseo pre-relaciones sexuales & $83.3 \%(\mathrm{Si})$ & $79.7 \%(\mathrm{Si})$ \\
Aseo post-relaciones sexuales & $82.5 \%(\mathrm{Si})$ & $79.7 \%(\mathrm{Si})$ \\
Tratamiento para salud sexual & $90 \%(\mathrm{No})$ & $91.9 \%(\mathrm{No})$ \\
Palpación en genitales & $50 \%(\mathrm{Si} / \mathrm{No})$ & $54.1 \%(\mathrm{No})$ \\
Dormir más de 8hrs & $\mathbf{8 0 \%}(\mathrm{No})$ & $\mathbf{6 3 . 5 \%}(\mathrm{No})$ \\
Dormir 3 a 7hrs & $81.7 \%(\mathrm{Si})$ & $70.3 \%(\mathrm{Si})$ \\
Sueño reparador & $61.7 \%(\mathrm{Si})$ & $59.5 \%(\mathrm{Si})$ \\
Cuesta conciliar el sueño & $63.3 \%(\mathrm{No})$ & $64.9 \%(\mathrm{No})$ \\
Actividad para conciliar sueño & $73.3 \%(\mathrm{No})$ & $89.2 \%(\mathrm{No})$ \\
Consumo medicamento & $91.7 \%(\mathrm{No})$ & $98.6 \%(\mathrm{No})$ \\
\hline
\end{tabular}

Nota: aspectos más sobresalientes sobre sexualidad y descanso, por institución, de los estudiantes universitarios participantes.

\section{Conclusión y discusión:}

Los resultados encontrados en este estudio permiten identificar una consistencia con los hallazgos encontrados con población de hombres universitarios en varios factores que son relativos a su autocuidado, porque se abarcan diferentes áreas de salud como puntos clave de factores de riesgo que pueden ser modificables.

Con respecto a la alimentación, el 59.2\% de los participantes de la Universidad Politécnica de Querétaro (UPQ), considera que se alimenta sanamente y en la Universidad Marista de Querétaro (UMQ) prevaleció el $50 \%$. En cuanto a la actividad física, el $57.8 \%$ y $56.8 \%$ respectivamente, realizan ejercicio más de 3 veces por semana. Esto no coincide con un estudio en donde se obtuvieron los siguientes datos: el $27 \%$ de los hombres dijo que su alimentación era buena, el 55\% regular y 18\% mala; con respecto a la actividad física, el 39\% de los hombres la realiza diariamente, 50\% cada semana y $11 \%$ no (Simon Olea, López Ampudia, Sandoval Cárdenas, \& Magaña Martínez, 2016).

En la automedicación se encontró que el 55.8\% en la UPQ y el 51.4\% en la UMQ la tienen como una práctica que se realiza con frecuencia. Con respecto a la exploración del cuerpo y específicamente en la palpación de pectorales, se obtuvo que el $71.7 \%$ de la UPQ y el $77 \%$ de la UMQ lo realizan. 
La palpación de genitales $50 \%$ y $54.1 \%$ respectivamente, no lo realizan. Relacionado con lo anterior, el estudio de Escobar (Escobar P \& Pico M, 2013) encontró un valor superior en esta práctica en sus participantes, ya que de los 152 hombres encuestados el 66.2\% tienen esta práctica siempre; con respecto a la exploración del cuerpo (lunares, manchas en la piel y masas en mama, testículos u otra parte del cuerpo), nuevamente se encuentra al $46.3 \%$ de los hombres no lo realizan.

En relación a los hábitos nocivos de los universitarios sobre el consumo de alcohol o tabaco, se obtuvo que el $80 \%$ de la UPQ y el $86.5 \%$ no fuman cigarros a diario, al igual que no consumen alcohol cada fin de semana el $68.3 \%$ y $51.4 \%$ respectivamente. En un estudio semejante, existe una ligera similitud en los porcentajes con respecto al consumo de tabaco, ya que se encontró que del total de alumnos abordados, $15 \%$ manifestaron que en la actualidad son fumadores, mientras que $85 \%$ no lo son; por su parte en el consumo de alcohol, este estudio no coincide, ya que existe un aumento en el porcentaje, ya que el $26 \%$ de los estudiantes se describieron como bebedores frecuentes, frente al $74 \%$ que se consideraron como bebedores muy ocasionales (sólo en ocasiones especiales) o no bebedores (Mantilla-Toloza, Villamizar, \& Peltzer, 2016).

En cuanto al área de la organización del sueño y el descanso, en la UPQ el $81.7 \%$ y en la UMQ el $73.5 \%$ duerme de entre 3 a 7 hrs., rubro muy amplio y que valdría la pena profundizar. De la misma manera, de ellos sólo el $61.7 \%$ de la UPQ y el $59.5 \%$ de la UMQ considera que su sueño fue reparador. Esto no coincide con los hallazgos de un estudio en donde se encuestaron a 155 universitarios, de los cuales 64 fueron hombres; el 58\% duerme al menos siete horas diarias, pero sólo el $36.8 \%$ duerme bien y se levanta descansado con frecuencia o siempre, habiendo un $85.2 \%$ que presenta somnolencia durante el día alguna veces, con frecuencia o siempre. Un 66.6\% trasnocha entre algunas veces y siempre, a un $66.5 \%$ le cuesta trabajo quedarse dormido y un $61.3 \%$ se despierta durante la noche en varias ocasiones con la misma frecuencia (Becerra, 2016).

La presente investigación muestra una aproximación al cuidado de la salud en los varones, de lo que se puede apreciar, de manera general, que existen diversas áreas de oportunidad en cuando a los aspectos en los que hay que orientarlos (alimentación, sueño, ejercicio, vida sexual), dar educación en salud y fortalecer estilos de vida saludables.

Por lo anterior, es de suma importancia que se realicen nuevos estudios de valoración del autocuidado en salud en varones universitarios, pues al ser un grupo de adultos jóvenes, estos tienen la oportunidad de cambiar el panorama del autocuidado en la actualidad.

Es importante concientizar a los varones universitarios sobre la importancia del autocuidado, ya que, de no ser así, las consecuencias pueden 
llegar a ser perjudiciales para la salud en el mediano y largo plazo, con la presencia de patologías agudas o crónicas o no previniendo a tiempo la aparición de las mismas.

\section{References :}

1. Becerra, S. (2016). Descripción de las conductas de salud en un grupo de estudiantes universitarios de Lima. Revista de Psicologia (Peru), 34(2), 239-260. https://doi.org/10.18800/psico.201602.001

2. Caballero D, C. C., Abello LI, R., \& Palacio S, J. (2018). Relación del burnout y el rendimiento académico con la satisfacción frente a los estudios en estudiantes universitarios. Avances En Psicología Latinoamericana, 25(2), 98-111. Retrieved from https://revistas.urosario.edu.co/index.php/apl/article/view/1208

3. Cámara de diputados del H. Congreso de la Unión. Reglamento de la Ley General de Salud en Materia de Investigaciones para la Salud., Ley General de Salud $\S$ (1987). Retrieved from http://www.cofepris.gob.mx/MJ/Paginas/Reglamentos.aspx

4. Comité Nacional de Bioética. Código de Nuremberg Normas éticas sobre experimentación en seres humanos, Comision Nacional de Bioética $\S$ (1947). Retrieved from http://www.conbioeticamexico.salud.gob.mx/descargas/pdf/normatividad/normatinternacion al/2.INTL._Cod_Nuremberg.pdf

5. CONAMED. Declaración de Helsinki de la Asociación Médica Mundial, 137 CONAMED $\S$ (2001). Retrieved from http://www.conamed.gob.mx/prof_salud/pdf/helsinki.pdf

6. Cubillas Rodríguez, M. J., Valdez, E. A., Domínguez Ibáñez, S. E., Román Pérez, R., Hernandez Montaño, A., \& Zapata Salazar, J. (2016). Creencias sobre estereotipos de género de jóvenes universitarios del norte de México. Diversitas: Perspectivas En Psicología, 12(2), 217-230. https://doi.org/10.15332/s17949998.2016.0002.04

7. da Cruz Mendonça, F. A., Magalhães Moreira, T. M., Leite Sampaio, L. R., Leite Brasil, B. M. B., Nobre de Arruda, C., Machado Diógenes, K. C. B., \& Guedes Braide, A. S. (2018). Associações entre o estilo de vida masculino e ações de promoção de saúde. Motricidade, 14(1), 355-361. Retrieved from http://www.scielo.mec.pt/pdf/mot/v14n1/v14n1a55.pdf

8. De Keijzer, B. (1997). El varón como factor de riesgo: masculinidad, salud mental y salud reproductiva. Tuñón, 15. Retrieved from http://www.sasia.org.ar/sites/www.sasia.org.ar/files/El varon como factor de riesgo.pdf

9. Escobar P, M. del P., \& Pico M, M. E. (2013). Autocuidado de la salud 
en jóvenes universitarios, Manizales, 2010-2011. Revista Facultad Nacional de Salud Pública, 31(2), 178-186. Retrieved from http://www.scielo.org.co/pdf/rfnsp/v31n2/v31n2a03.pdf

10. Estrada-Durand, P., \& Salinas-Salas, C. (2019). Consumo de Alcohol como factor sociado a La depresión en estudiantes varones de Medicina Humana de la Universidad Ricardo Palma del Año 2018. Revista de La Facultad de Medicina Humana, 19(1), 1-5. https://doi.org/10.25176/rfmh.v19.n1.1801

11. Franco Peláez, Z. R., \& Duque Escobar, J. A. (2015). La bioética y el autocuidado de la salud: imperativos para la formación integral en la universidad. Acta Bioethica, 21(1), 37-44. https://doi.org/10.4067/S1726-569X2015000100005

12. Guerrero-Wyss, M., Méndez, A., Gajardo, F., \& Durán Agüero, S. (2018). Relación de hábito tabáquico, estado nutricional y calidad de sueño en estudiantes de la Universidad San Sebastián. Latinoamericana de Hipertensión, 13(1), 43-48. Retrieved from http://www.revhipertension.com/rlh_1_2018/relacion_habito_tabaqui co.pdf

13. Mantilla-Toloza, S. C., Villamizar, C. E., \& Peltzer, K. (2016). Consumo de alcohol, tabaquismo y características sociodemográficas en estudiantes universitarios. Universidad y Salud, 18(1), 7. https://doi.org/10.22267/rus.161801.14

14. OMS. Constitución de la Organización Mundial de la Salud (1946). Retrieved from https://apps.who.int/gb/bd/PDF/bd47/SP/constitucionsp.pdf?ua $=1$

15. Pinto, A. D., Aratangy, T., Abramovich, A., Devotta, K., Nisenbaum, R., Wang, R., \& Kiran, T. (2019). Routine collection of sexual orientation and gender identity data: A mixed-methods study. Cmaj, 191(3), E63-E67. https://doi.org/10.1503/cmaj.180839

16. Quinchiguano Caraguay, Y. S. (2017). Autocuidado en los estudiantes de la Unidad Educativa "Pio Jaramillo Alvarado" Loja 2016-2017. Universidad Nacional de Loja. Retrieved from https://dspace.unl.edu.ec/jspui/handle/123456789/18670?mode=simp le

17. Sabó, D. (2000). Comprender la salud de los hombres. Un enfoque relacional y sensible al género. Harvard Center for Population and Development Studies.

18. Simon Olea, L. N., López Ampudia, M. G., Sandoval Cárdenas, R. I., \& Magaña Martínez, B. (2016). Nivel de Autocuidado en Estudiantes Universitarios de Cinco Licenciaturas del Área de la Salud. Revista CuidArte, $5(10)$, 32. https://doi.org/10.22201/fesi.23958979e.2016.5.10.69114 
19. Uribe J, T. M. (1999). El autocuidado y su papel en la promoción de la salud. Investigacion y Educacion En Enfermeria. Retrieved from file:///C:/Users/HP/Downloads/Dialnet-

ElAutocuidadoYSuPapelEnLaPromocionDeLaSalud-5331981.pdf 